University of Nebraska - Lincoln

DigitalCommons@University of Nebraska - Lincoln

Timothy J. Gay Publications

Research Papers in Physics and Astronomy

September 1997

\title{
Electron-Collision-Induced Alignment of Rare Gases near Threshold
}

V. Zeman

Drake University, Des Moines, lowa

K. Bartschat

Drake University, Des Moines, lowa

Timothy J. Gay

University of Nebraska - Lincoln, tgay1@unl.edu

K. W. Trantham

Drake University, Des Moines, lowa

Follow this and additional works at: https://digitalcommons.unl.edu/physicsgay

Part of the Physics Commons

Zeman, V.; Bartschat, K.; Gay, Timothy J. ; and Trantham, K. W., "Electron-Collision-Induced Alignment of Rare Gases near Threshold" (1997). Timothy J. Gay Publications. 29.

https://digitalcommons.unl.edu/physicsgay/29

This Article is brought to you for free and open access by the Research Papers in Physics and Astronomy at DigitalCommons@University of Nebraska - Lincoln. It has been accepted for inclusion in Timothy J. Gay Publications by an authorized administrator of DigitalCommons@University of Nebraska - Lincoln. 


\title{
Electron-Collision-Induced Alignment of Rare Gases near Threshold
}

\author{
V. Zeman and K. Bartschat \\ Department of Physics and Astronomy, Drake University, Des Moines, Iowa 50311 \\ T. J. Gay and K. W. Trantham* \\ Behlen Laboratory of Physics, University of Nebraska, Lincoln, Nebraska 68588-0111
}

(Received 30 May 1997)

\begin{abstract}
Results of a semirelativistic $R$-matrix calculation are compared with experimental values for light polarizations and alignment parameters of the second excited-state manifolds of neon and krypton following electron impact excitation. The calculations focus on the near-threshold regime, where negative-ion resonances have a significant effect on the atomic alignment. A grouping of the alignment values according to the electronic angular momentum $J$ of the excited state is observed and qualitatively explained by angular momentum coupling considerations. [S0031-9007(97)04027-1]
\end{abstract}

PACS numbers: $34.80 . \mathrm{Dp}, 34.80 . \mathrm{Nz}$

When atoms are excited by beams of electrons, they generally emit polarized light. The study of this polarization can reveal information about the collision dynamics unavailable from measurements of the collision cross sections alone [1]. Studies with spin-polarized incident electrons can provide even more detailed information about the collision physics [2]. Increasingly sophisticated and detailed measurements, in concert with the advent of new theoretical methods and dramatic improvements in desktop computing power in the last decade, have placed our understanding of electronic collisions with the simplest ground-state atoms such as $\mathrm{H}, \mathrm{He}$, and the light alkalis on a firm footing [3]. Our knowledge of electronic collisions involving heavy atoms with many outer-shell electrons, in which relativistic effects can be important, is much poorer. Early attempts to cobble together relativistic theories of electron-heavy-atom collisions have been severely limited by computing power, and have met with only limited success [3].

In this Letter, we use results from state-of-the-art semirelativistic $R$-matrix calculations, which do not suffer as much from difficulties endemic to earlier perturbative and close-coupling approaches, to provide new physical insight into one of the oldest problems in electron-atom scattering: collision-induced alignment of the excited state in an axially symmetric geometry, i.e., without the detection of the scattered electrons [4]. This subject has been the focus of recent attention [5-9].

One of the central issues in this problem has been the near-threshold behavior of the alignment (and hence the fluorescence polarization), which is complicated by the formation of negative-ion resonances in the threshold region [6,8-12]. In this regime perturbative calculations are clearly inappropriate. On the other hand, it is crucial that close-coupling approaches have an adequate basis set to describe resonance formation and decay. It is in this energy range, within $5 \mathrm{eV}$ of threshold, that we focus our discussion. Our method is particularly powerful here because of its inclusion of a large number of coupled channels. As we will show, spin-dependent relativistic effects, which can be probed experimentally using spin-polarized electrons, and which have historically been difficult to predict theoretically, are well described by our calculations. By comparing the alignment values for a complete manifold of excited states, a systematic grouping of alignment by $J$ value is revealed and explained using angular momentum arguments.

Studies of electron-collision-induced alignment in heavy atoms have, to date, been cursory. (We do not consider here experiments in which the scattered electron and the decay photon are detected in coincidence.) The pioneering measurements of Skinner and Appleyard, made in 1927, involved $\mathrm{Hg}$ targets [4]. More recently, experiments have been performed with alkalis and alkali earths, noble gases, and with a few other targets such as the group IIB elements [11]. With the exception of the work of the Münster group on $\mathrm{Hg}$ [13], none involved polarized electrons. Furst et al. [5,14] made the first measurements using a polarized electron beam in the study of the heavy noble gases, and the Perth group has recently reported extensive measurements with neon using polarized electrons as well [7-9].

Heavy noble gases have several advantages as targets. Perhaps most importantly, they present a stringent challenge to theory. The $n p^{5}(n+1) p$ excited state configuration provides a complex manifold of ten fine-structure levels. This allows for a detailed comparison of excitation dynamics for different final-state spin-orbit couplings. In addition, noble gases are easy to handle experimentally, and the $n p^{5}(n+1) p$ manifold decays primarily by visible radiation, making the photon polarimetry relatively easy. The apparatuses used for our measurements have been described earlier $[5,15]$.

The details of the $R$-matrix calculation for $e$-Ne scattering will be presented elsewhere [16]. Briefly, it is a 31-state semirelativistic model, based on the Belfast $R$-matrix codes [17], where the ground state and all excited states with configurations $2 p^{5} 3 s, 2 p^{5} 3 p, 2 p^{5} 4 s$, and 
$2 p^{5} 3 d$ are included in the close-coupling expansion. In addition, special care has been taken to produce a $3 p$ orbital that is particularly suitable for the description of the states of interest. This sophisticated basis set, in combination with the inclusion of the one-electron relativistic BreitPauli terms in the Hamiltonian, gives us confidence in the reliability of our results for excitation of the heavy noble gases in the near-threshold region. The present calculation represents the first step towards a converged " $R$-matrix with pseudostates" $[18,19]$ (RMPS) treatment of this complicated problem which, following the RMPS' success for light nonrelativistic targets, would be highly desirable at intermediate energies.

In the electric-dipole approximation, two linear polarization fractions, $P_{1}$ and $P_{2}$, are required to describe the alignment of the atomic excited state. These correspond to polarization along $\left(P_{1}\right)$, or at $45^{\circ}\left(P_{2}\right)$, to the electron beam axis. Equivalently, they describe the magnitude and direction of the atomic alignment, or the second moment of the electronic distribution.

Figure 1 shows $P_{1}$ values for excitation of the $3 p[5 / 2]_{3}\left({ }^{3} D_{3}\right)$ and $3 p^{\prime}[3 / 2]_{2}$ (" ${ }^{3} P_{2}$ ") states in neon. (The quotation marks indicate that the latter is not a true Russell-Saunders state.) These data were taken with photons observed at a polar angle of $\theta=135^{\circ}$. First, we note that the theory does a good qualitative job of describing the energy dependence and magnitude of the data. This is noteworthy in itself given the complexity of the physical process and the target under consideration. (Cascading from higher levels begins to be appreciable about $2 \mathrm{eV}$ above threshold; this may account for the increasing discrepancy between theory and experiment at higher energies.) Second, the ${ }^{3} D_{3}$ data approach the required kinematic threshold value of $P_{1}=0.28$ [15]. This value is determined for a well $L S$-coupled ${ }^{3} D$ state by the fact that (i) it must be excited by exchange and (ii) only $M_{L}=0$ magnetic sublevels can be populated at threshold. Since the ${ }^{3} P_{2}$ level is not well $L S$ coupled, it does not have a kinematically defined threshold value. The calculation also indicates that negative-ion resonances

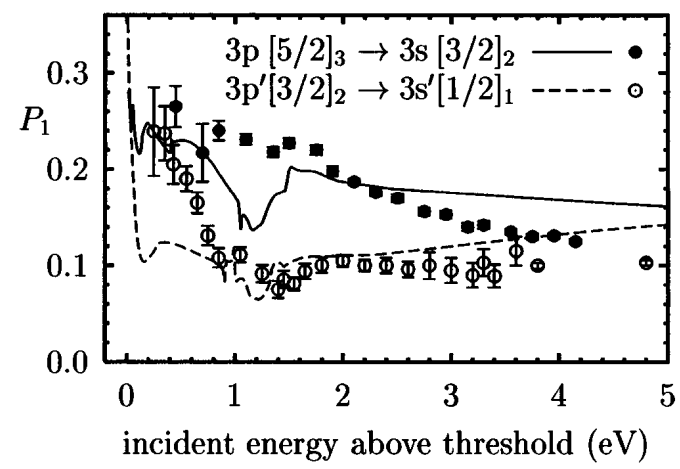

FIG. 1. Linear polarization $P_{1}$ after electron impact excitation of Ne. The experimental data were taken at a polar angle relative to the incident beam axis $\theta=135^{\circ}$.
[6,12], which decay into the ${ }^{3} D_{3}$ state, should affect $P_{1}$. These are not seen as clearly in the experimental data because of the energy width of the electron beam we used $(\approx 0.3 \mathrm{eV})$.

Qualitatively, the most interesting aspect of the ${ }^{3} D_{3}$ data is the fact that they do not drop as rapidly from their threshold value as do the ${ }^{3} P_{2}$ results. Our calculations indicate that the ${ }^{3} P_{2}$ values of $P_{1}$ drop to less than $50 \%$ of their threshold value within $0.1 \mathrm{eV}$ of threshold. (The qualitative differences between theory and experiment below $1 \mathrm{eV}$ for this state are due, at least in part, to the high energy "tail" of our electron beam.) In contrast, the calculated ${ }^{3} D_{3}$ values do not decrease to half their initial value until $6 \mathrm{eV}$ above threshold. An interesting difference between the ${ }^{3} D_{3}$ and ${ }^{3} P_{2}$ states concerns the angular momentum $j_{c}$ of their respective cores. While they both have their excited-electron orbital angular momentum, their excitedelectron spins, and $j_{c}$ "lined up" to give the maximal $J$ for the state, the ${ }^{3} D_{3}$ state has an alignable $\left(j_{c}=3 / 2\right)$ core while the ${ }^{3} P_{2}$ state does not $\left(j_{c}=1 / 2\right)$. The tendency of the atomic ${ }^{3} D_{3}$ alignment to remain high well above threshold has thus in the past been interpreted in terms of an alignment "flywheel" model $[7,20]$ : the "storage" of alignment in the ${ }^{3} D_{3}$ core could reduce both the depolarizing influence of negative-ion decay and the ordinary fall of $P_{1}$ from its threshold value as sublevels with $M_{L}>0$ begin to be excited.

This type of physical information, made apparent by the comparison of states with different angular momentum coupling schemes in the same manifold, points out the utility of such comparative studies. Consequently, we now look more broadly at the dynamical parameter that uniquely determines the value of $P_{1}$ : the relative alignment

$$
\left\langle t(J)_{20}^{+}\right\rangle \equiv \frac{\left\langle T(J)_{20}^{+}\right\rangle}{\left\langle T(J)_{00}^{+}\right\rangle} .
$$

The integrated state multipoles are defined by [21]

$$
\begin{aligned}
\left\langle T(J)_{K Q}^{\dagger}\right\rangle= & \sum_{M^{\prime} M}(-1)^{J-M^{\prime}} \sqrt{2 K+1} \\
& \times\left(\begin{array}{ccc}
J & J & K \\
M^{\prime} & -M & -Q
\end{array}\right)\left\langle J M^{\prime}|\rho| J M\right\rangle .
\end{aligned}
$$

Here $\left\langle J M^{\prime}|\rho| J M\right\rangle$ is an element of the density matrix that describes the excited atomic state if the scattered electrons are not observed. The value of $P_{1}$ is independent of incident electron polarization and, for dipole emission from a state with angular momentum $J$ to one with $J_{f}$, can be expressed as [21]

$$
P_{1}=\frac{3\left\{\begin{array}{c}
112 \\
J J J_{f}
\end{array}\right\}\left\langle t_{20}^{+}\right\rangle \sin ^{2} \theta}{\frac{(-1)^{J+J_{f}}}{G_{2}} \sqrt{\frac{8}{3(2 J+1)}}-\left\{\begin{array}{c}
112 \\
J J J_{f}
\end{array}\right\}\left\langle t_{20}^{+}\right\rangle\left(3 \cos ^{2} \theta-1\right)},
$$


where $G_{2}$ is a factor to account, if necessary, for depolarization of the radiation due to atomic hyperfine structure.

Figure 2 shows results for $\left\langle t_{20}^{+}\right\rangle$as a function of incident electron energy for the eight $\mathrm{Ne}$ states with configuration $2 p^{5} 3 p$ and $J \neq 0$. (For $J=0,\left\langle t_{20}^{+}\right\rangle$is identically zero.) For energies of only a few tenths of an $\mathrm{eV}$ above threshold, we note a striking systematic effect: the results cluster by $J$ value of the excited state. Although the agreement between theory and experiment (and between different sets of experimental data) is not perfect, the general trend is clearly confirmed.

We can qualitatively explain this clustering with arguments based on angular momentum coupling. Since the diagonal elements of the density matrix are the angleintegrated magnetic sublevel cross sections $Q_{M}$, the relative alignment parameters for the various $J$ values can be written as [21]:

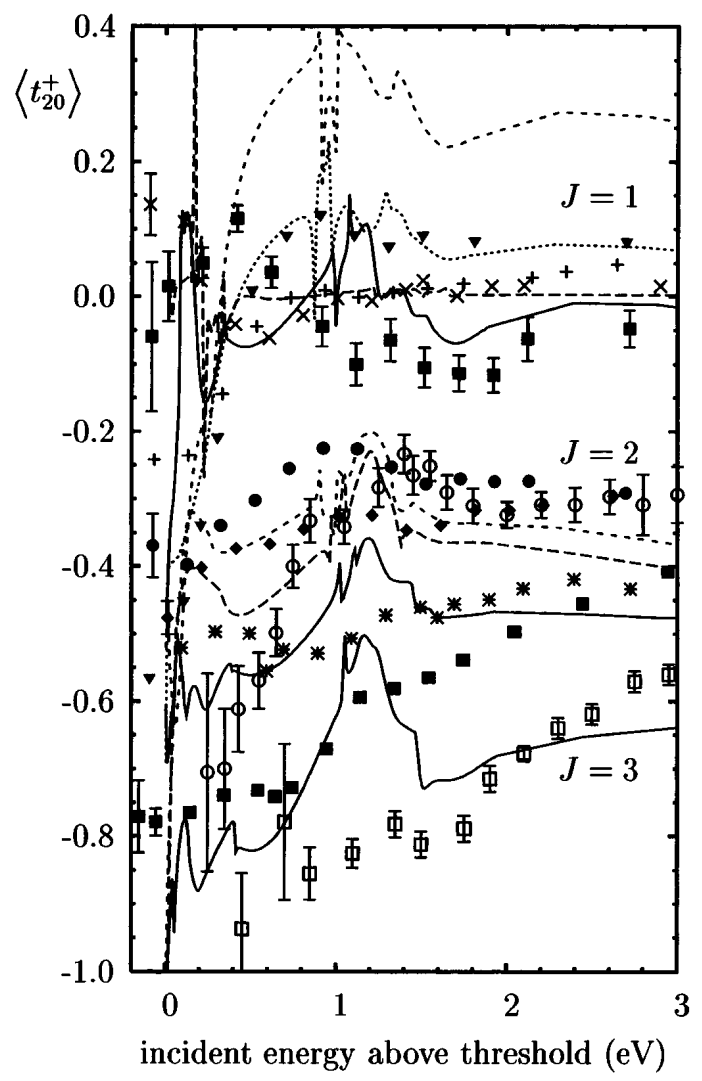

FIG. 2. Relative alignment parameter $\left\langle t_{20}^{+}\right\rangle$after electron impact excitation of the $\mathrm{Ne} 2 p^{5} 3 p$ manifold. For incident energies more than $0.2 \mathrm{eV}$ above threshold, the top four curves belong to states with $J=1$, the next three to $J=2$, and the bottom one to the $J=3$ state. In detail, the curves and symbols are as follows: $J=1$ : solid line, $\times, 3 p[3 / 2]_{1}$; long dashes, squares, $3 p[1 / 2]_{1}$; short dashes, triangles, $3 p^{\prime}[3 / 2]_{1}$; dots,,$+ 3 p^{\prime}[1 / 2]_{1}$. $J=2$ : solid line, *, $3 p[5 / 2]_{2}$; long dashes, diamonds, $3 p[3 / 2]_{2}$; short dashes, $\bullet$ and $\circ, 3 p^{\prime}[3 / 2]_{2} . \quad J=3$ : solid line, open, and solid squares, $3 p[5 / 2]_{3}$. The experimental data are taken from $\mathrm{Yu}$ et al. $[8,9]$ and from this work ( $\square$ for $3 p[5 / 2]_{3}$ and $\circ$ for $\left.3 p^{\prime}[3 / 2]_{2}\right)$.

$$
\begin{gathered}
J=1:\left\langle t_{20}^{+}\right\rangle=\sqrt{2} \frac{Q_{1}-Q_{0}}{Q_{1}+Q_{0}} ; \\
J=2:\left\langle t_{20}^{+}\right\rangle=\sqrt{\frac{10}{7} \frac{2 Q_{2}-Q_{1}-Q_{0}}{2 Q_{2}+2 Q_{1}+Q_{0}}} ; \\
J=3:\left\langle t_{20}^{+}\right\rangle=\frac{1}{\sqrt{3}} \frac{5 Q_{3}-3 Q_{1}-2 Q_{0}}{2 Q_{3}+2 Q_{2}+2 Q_{1}+Q_{0}} .
\end{gathered}
$$

Furthermore, conservation of the total angular momentum of the collision system implies the selection rule

$$
M+m_{\ell_{f}}+m_{f}=m_{i},
$$

where $m_{\ell_{f}}$ is the orbital angular momentum component of the scattered electron while $m_{f}\left(m_{i}\right)$ is its final (initial) spin projection with respect to the quantization axis. Note that Eq. (7) holds for an initial atomic state with $J=0$ and our choice of quantization axis along the incident beam direction.

It follows from Eq. (7) that excitation processes without spin flip $\left(m_{f}=m_{i}\right)$ require $M=-m_{\ell_{f}}$, while excitation processes with spin flip $\left(m_{f}=-m_{i}\right)$ require $M=-m_{\ell_{f}} \pm 1$. Consequently, there are no contributions from projectile partial waves with $\ell_{f}=0,1$ to $Q_{3}$ at all, while $Q_{2}$ contains only an exchange contribution from $\ell_{f}=1$. On the other hand, optically forbidden transitions, like the ones discussed in this work, are strongly affected by partial waves with small angular momenta. Hence one can expect $Q_{0}$ and $Q_{1}$ to be significantly larger than $Q_{2}$ and $Q_{3}$, respectively, especially near threshold. According to Eqs. (4)-(6), this result will indeed lead to a large negative alignment for states with $J \geq 2$. On the other hand, the alignment parameter for the $J=1$ states contains the difference between $Q_{1}$ and $Q_{0}$ in the numerator, and so one would expect a smaller value in this case. This grouping of alignment by $J$ has not been observed before because of the lack of comprehensive data sets for a given atom, and because much of the previous alignment data was taken with unresolved fine structure.

When transversely polarized incident electrons are used in these experiments, it is possible to also produce alignment corresponding to a tilting of the charge cloud away from the electron beam axis in the plane perpendicular to the direction of electron polarization. Such tilting is described by the relative alignment parameter $\left\langle t_{21}^{+}\right\rangle$. It can occur only if exchange excitation occurs and either (a) appreciable spin-orbit forces act on the continuum electron ("Mott scattering") or (b) the excited state is not well $L S$ coupled $[14,22]$. For noble gases lighter than radon, we have shown previously that Mott scattering is negligible [14]. Hence any nonzero $P_{2}$ values must be due to the breakdown of $L S$ coupling in the excited state. The production of nonzero $\left\langle t_{21}^{+}\right\rangle$thus requires a combination of exchange and internal relativistic effects. As such, it provides a stringent test of any theoretical calculation $[9,22]$.

Values of $P_{2}$ for several transitions in $\mathrm{Ne}$ and $\mathrm{Kr}$ are shown in Fig. 3. (The $e-\mathrm{Kr}$ calculation was set up similarly to the one for $\mathrm{Ne}[16]$.) For both targets the agreement between theory and experiment is very satisfactory. 

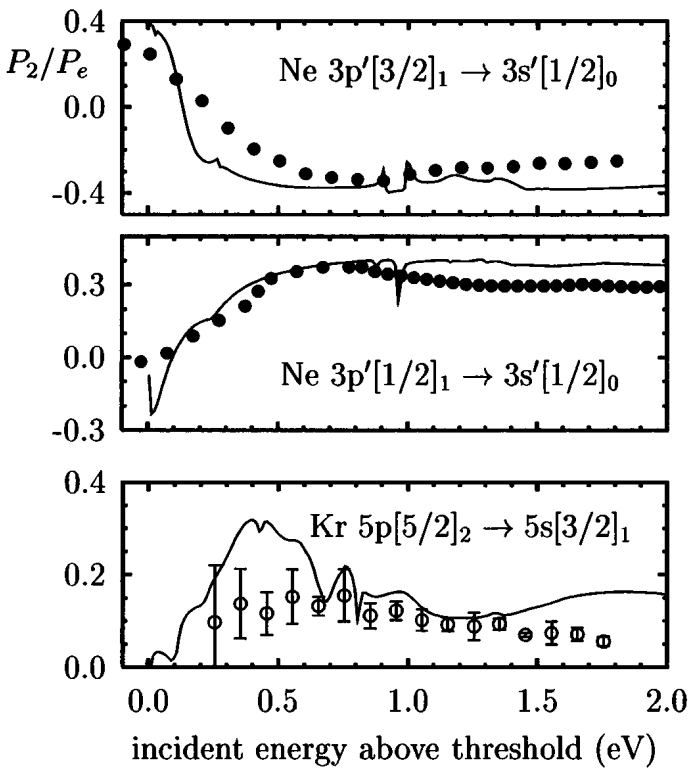

FIG. 3. Linear polarization $P_{2}$ of $\mathrm{Ne}$ and $\mathrm{Kr}$ transitions measured at $\theta=90^{\circ}$, normalized to $100 \%$ incident electron polarization $P_{e}$. The experimental data for $\mathrm{Ne}$ and $\mathrm{Kr}$ are from Refs. [9] and [5], respectively.

Note that the sign of the published experimental results [9] for $e$-Ne has been reversed, since a sign error has just been confirmed [23]. We expect that the remaining discrepancies between theory and experiment for the $P_{2}$ parameter in $\mathrm{Kr}$ are mostly due to the fact that the structure calculation in $\mathrm{Kr}$ is more difficult and that the use of nonrelativistic one-electron orbitals may no longer be appropriate.

In summary, we have presented a sophisticated numerical calculation for electron impact excitation of $\mathrm{Ne}$ and $\mathrm{Kr}$, and have compared the results with recent experimental data for the fluorescence polarization and atomic alignment. The satisfactory agreement obtained between theory and experiment gives us confidence in the interpretation of the complete set of alignment parameters $\left\langle t_{20}^{+}\right\rangle$for the $2 p^{5} 3 p$ multiplet in Ne. The interesting grouping of the results according to the total electronic angular momentum $J$ of the excited state can be qualitatively explained using angular momentum coupling rules and, therefore, is expected to be a general feature for similar excitation processes in $\mathrm{Ar}, \mathrm{Kr}$, and $\mathrm{Xe}$. While some evidence for the validity of the "flywheel model," i.e., the storage of alignment in the core of the excited atom was found, any such effect is not as important as the overall dynamical clustering with $J$. In light of the present findings, further joint experimental and theoretical efforts to fully understand the electron impact excitation of all the heavy noble gases seem very desirable.

This work was supported by the National Science Foundation under Grants No. PHY-9605124 (V.Z. and
K. B.) and No. PHY-9504350 (T. J. G. and K. W. T.). We are grateful to D. H. Yu, P. A. Hayes, and J.F. Williams for making available their data in numerical form prior to publication. T.J.G. also acknowledges stimulating discussions with U. Fano and J. E. Furst.

*Present address: Electron Physics Group, Australian National University, Canberra ACT, Australia.

[1] N. Andersen, J. W. Gallagher, and I. V. Hertel, Phys. Rep. 165, 1 (1988).

[2] N. Andersen and K. Bartschat, Adv. At. Mol. Phys. 36, 1 (1996).

[3] N. Andersen, K. Bartschat, J. T. Broad, and I. V. Hertel, Phys. Rep. 279, 251 (1997), and references therein.

[4] H.W. B. Skinner and E.T.S. Appleyard, Proc. R. Soc. London A 117, 224 (1927).

[5] J. E. Furst, W. M. K. P. Wijayaratna, D. H. Madison, and T. J. Gay, Phys. Rev. A 47, 3775 (1993).

[6] C. Norén, W. L. Karras, J. W. McConkey, P. Hammond, and K. Bartschat, Phys. Rev. A 53, 1559 (1996); 53, 3253 (1996); 54, 510 (1996).

[7] P. A. Hayes, D. H. Yu, J.E. Furst, M. Donath, and J. F. Williams, J. Phys. B 29, 3989 (1996).

[8] D. H. Yu, P.A. Hayes, J.F. Williams, and J.E. Furst, J. Phys. B 30, 1799 (1997).

[9] D. H. Yu, P.A. Hayes, J.E. Furst, and J.F. Williams, Phys. Rev. Lett. 78, 2724 (1997).

[10] R. H. McFarland and M.H. Mittleman, Phys. Rev. Lett. 20, 899 (1968).

[11] D. W. O. Heddle and J. W. Gallagher, Rev. Mod. Phys. 61, 221 (1989); D. W. O. Heddle, Contemp. Phys. 17, 443 (1976), and references therein.

[12] S. J. Buckman and C. W. Clark, Rev. Mod. Phys. 66, 539 (1994).

[13] A. Wolcke, K. Bartschat, K. Blum, H. Borgmann, G. F. Hanne, and J. Kessler, J. Phys. B 16, 639 (1983).

[14] J.E. Furst, T.J. Gay, W.M. K.P. Wijayaratna, K. Bartschat, H. Geesmann, M. A. Khakoo, and D. H. Madison, J. Phys. B 25, 1089 (1992).

[15] K. W. Trantham, T. J. Gay, and R. J. Vandiver, Rev. Sci. Instrum. 67, 4103 (1996).

[16] V. Zeman and K. Bartschat (to be published).

[17] K. A. Berrington, W.B. Eissner, and P.H. Norrington, Comput. Phys. Commun. 92, 290 (1995).

[18] K. Bartschat, E. T. Hudson, M.P. Scott, P. G. Burke, and V. M. Burke, J. Phys. B 29, 115 (1996).

[19] K. Bartschat, E. T. Hudson, M.P. Scott, P. G. Burke, and V. M. Burke, Phys. Rev. A 54, R998 (1996).

[20] K. W. Trantham, M. E. Johnston, and T. J. Gay, Bull. Am. Phys. Soc. 41, 1135 (1996).

[21] K. Blum, Density Matrix Theory and Applications (Plenum, New York, 1996), 2nd ed.

[22] K. Bartschat and K. Blum, Z. Phys. A 304, 85 (1982).

[23] D. H. Yu, P. A. Hayes, and J. F. Williams (private communication). 